\title{
Non-ideal SSS Coherent Detection in 3GPP LTE System
}

\author{
You Zhou, Hanying Hu, Ping Wang \\ Zhengzhou Information Technology Institute \\ Zhengzhou, China \\ emailzhouyou@sina.com
}

\begin{abstract}
In the LTE system, SSS coherent detection is one of the commonly used methods. However, Theoretical analysis of the performance of non-ideal coherent detection is rarely seen. Properly modeling the Doppler Effect and channel estimation error, the paper focuses on the mathematic deduction of nonideal SSS coherent detection performance, which was approved by simulations. SSS coherent detection performance in TDD/FDD systems is compared with non-coherent detection methods under various assumptions of channel estimation error, which provides a basis for choosing SSS detection methods in a practical system.
\end{abstract}

Keywords-LTE; SSS; coherent detection; non-coherent detecton; non-ideal

\section{INTRODUCTION}

The $3^{\text {rd }}$ Generation Partnership Project (3GPP) Long Term Evolution (LTE) is the key technology of the next generation of cellular mobile communication system [1][2]. User equipment (UE) acquires the cell timing, frequency synchronization and cell identification information through a cell search process [3]. A two-step cell search is adopted in the LTE [2]: firstly, time slot synchronization and the sector identification of the cell are acquired by detecting the primary synchronization signal (PSS). Secondly, frame synchronization and cell group identification are acquired by detecting the secondary synchronization signal (SSS).

SSS detection methods are divided into two categories: coherent detection and non-coherent detection. In the coherent detection, channel estimation through PSS is treated as the one of SSS [4]. However, Doppler Effect and channel estimation error make the detection performance worse. In [5], the influence of channel on SSS is eliminated through zero forcing method, which may magnify the noise and deteriorate the detection performance. Non-coherent detection takes advantage of the good self-correlation and cross-correlation properties of SSS in the frequency domain. In [6], segment correlation is performed to combat the frequency selective fading at the cost of bringing down the SSS self-correlation and cross-correlation performance. In [7], it assumes that channel frequency responses of adjacent subcarriers are highly correlated and eliminates the influence of channel by difference operation, which may reduce the SNR.

[6]-[8] have pointed out that the SSS coherent detection performance drops through simulation methods. The influence of channel estimation error on the coherent detection performance is rarely mentioned. Properly modeling the Doppler Effect and channel estimation error, mathematic deduction of non-ideal SSS coherent detection performance is given in the paper. The theoretical result is approved by simulations. SSS theoretical and simulated coherent detection performance with different channel estimation error in TDD/FDD systems are given and compared with that of non-coherent detection, which provides a basis for the selection of detection algorithm in a practical system.

The remainder of this paper is organized as follows. A brief introduction about SSS and a system model are given in Section II. Section III gives the coherent detection structure and Section IV deduces the theoretical coherent detection performance. In section $\mathrm{V}$, the performance of the coherent detection structure under different circumstances is simulated and compared with that of theoretical result. Finally the conclusions are drawn in Section VI.

\section{SYSTEM DESCRIPTION}

A. SSS

PSS and SSS occupy the central 6 Resource Blocks (RB) of the system bandwidth [2]. In a FDD system, PSS and SSS are mapped to the last and penultimate OFDM symbols in slots 0 and 10 respectively. In a TDD system, PSS is mapped to the third OFDM symbol in subframes 1 and 6, SSS is mapped to last OFDM symbol in slots 1 and 11 . Because of the Doppler Effect, the time space between PSS and SSS deteriorates the coherent detection performance, especially of the FDD system, in which the space is much bigger.

SSS is determined by 168 cell group identifications and 3 sector identifications [2]:

$$
\begin{array}{r}
d(2 n)= \begin{cases}s_{0}^{\left(m_{0}\right)}(n) c_{0}(n), & \text { subframe0 } \\
s_{1}^{\left(m_{1}\right)}(n) c_{0}(n), & \text { subframe5 }\end{cases} \\
d(2 n+1)= \begin{cases}s_{1}^{\left(m_{1}\right)}(n) c_{1}(n) z_{1}^{\left(m_{0}\right)}(n), & \text { subframe0 } \\
s_{0}^{\left(m_{0}\right)}(n) c_{1}(n) z_{1}^{\left(m_{1}\right)}(n), & \text { subframe5 }\end{cases}
\end{array}
$$

where $0 \leq n \leq 30 . s_{0}^{\left(m_{0}\right)}(n), s_{1}^{\left(m_{1}\right)}(n), z_{1}^{\left(m_{0}\right)}(n)$ and $z_{1}^{\left(m_{1}\right)}(n)$ are cell group identification relevant scrambling sequences. $c_{0}(n)$ and $c_{1}(n)$ are sector identification related scrambling sequences. The paper assumes that the first step of the cell search has been accomplished; cell slot synchronization and sector identification have been acquired. 


\section{B. System Model}

The received SSS signal in the frequency domain can be expressed as:

$$
\mathbf{Y}=\mathbf{H d}_{t, f}+\mathbf{N}
$$

where $\mathbf{H}=\operatorname{diag}\left\{H_{0}, H_{1}, \ldots, H_{61}\right\}$ represents the frequency domain channel attenuation matrix, $\mathbf{d}_{t, f}=\left[d_{t, f, 0}, d_{t, f, 1}\right.$ $\left., \ldots, d_{t, f, 61}\right]^{T}$ is the SSS frequency signal with cell group identification $t \in\{0,1, \ldots, 167\}$ in the subframe $f \in\{0,5\}$. $\mathbf{N}$ is a vector of additive white Gaussian noise with $\mathbf{N} \sim \mathcal{C N}\left(\mathbf{0}, \sigma^{2} \mathbf{I}\right)$.

\section{COHERENT DETECTION}

Coherent detection structure is shown in Figure 1. Firstly, the received frequency SSS sequence $\mathbf{Y}$ is divided into $\mathbf{Y} 0=\left[y_{0}, y_{2}, \ldots, y_{60}\right]^{T}$ and $\mathbf{Y} 1=\left[y_{1}, y_{3}, \ldots, y_{61}\right]^{T}$ according to their odd-even relationships. Then the scrambling item in the received signal is eliminated by $c_{0}(n)$ and $c_{1}(n)$ generated from the sector identification, which is acquired by PSS detection in the first step of cell search:

$$
\left\{\begin{array}{l}
\mathbf{Z} 0=\mathbf{Y} 0 . * \mathbf{c}_{0}=\mathbf{s}_{a}^{m_{a}}+\mathbf{N} 1 \cdot * \mathbf{c}_{0}=\mathbf{s}_{a}^{m_{a}}+\mathbf{N} 3 \\
\mathbf{Z} 1=\mathbf{Y} 1{ }^{*} \mathbf{c}_{1}=\mathbf{s}_{b}^{m_{b}} \cdot * \mathbf{z}_{1}^{m_{a}}+\mathbf{N} 2 * \mathbf{c}_{1}=\mathbf{s}_{b}^{m_{b}} \cdot \mathbf{z}_{1}^{m_{a}}+\mathbf{N} 4
\end{array}\right.
$$

where “*” represents corresponding item multiplication, and $\quad \mathbf{N} 1=\left[N_{0}, N_{2}, \ldots, N_{60}\right]^{T}, \quad \mathbf{N} 2=\left[N_{1}, N_{3}, \ldots, N_{61}\right]^{T}$, $\mathbf{c}_{0}=\left[c_{0}(0), c_{0}(1), \ldots, c_{0}(31)\right]^{T}, \quad \mathbf{c}_{1}=\left[c_{1}(0), c_{1}(1), \ldots, c_{1}(31)\right]^{T}$. Since the frame synchronization has not yet been accomplished, it is uncertain about the index of the current received subframe. So there is no certainty about $a$ and $b$, however, it is certain that $a \in\{0,1\}, b \in\{0,1\}, a \neq b$. Because the value of each item of $\mathbf{c}_{0}$ and $\mathbf{c}_{1}$ is 1 or -1 , so statistical property of $\mathbf{N} 3=\mathbf{N} 1{ }^{*} \mathbf{c}_{0}$ and $\mathbf{N} 4=\mathbf{N} 2 .{ }^{*} \mathbf{c}_{1}$ remains the same. $m_{a}$ can be detected via $\mathbf{Z} 0$ :

$$
\begin{aligned}
\hat{m}_{a} & =\underset{m}{\arg \min }\left\|\operatorname{Re}\left(\mathbf{Z} 0-\left(\mathbf{s}^{m}\right)^{T} \hat{\mathbf{H}}_{0}\right)\right\|^{2} \\
& =\underset{m}{\arg \min } \sum_{i=0}^{31}\left\{\operatorname{Re}\left[\mathbf{Z} 0_{i}-\mathbf{s}_{i}^{m} \hat{\mathbf{H}}_{0, i}\right]\right\}^{2}, m \in\{0,1, \ldots 30\}
\end{aligned}
$$

where $\operatorname{Re}(*)$ represents the real component of a complex number, $\hat{\mathbf{H}}_{0}=\left[\hat{H}_{0}, \hat{H}_{2}, \ldots, \hat{H}_{60}\right]^{T}$ is the channel estimation vector. $\mathbf{Z} 1$ is descrambled with $\mathbf{z}_{1}^{\left(\hat{m}_{a}\right)}$ generated from $\hat{m}_{a}$, then $\hat{m}_{b}$ is obtained through a coherent method:

$$
\hat{m}_{b}=\underset{m}{\arg \min } \sum_{i=0}^{30}\left\{\operatorname{Re}\left[\mathbf{z}_{1, i}^{\left(\hat{m}_{a}\right)} \mathbf{Z} 1_{i}-\mathbf{s}_{i}^{m} \hat{\mathbf{H}}_{1, i}\right]\right\}^{2}, m \in\{0,1, \ldots 30\}
$$

where $\hat{\mathbf{H}}_{1}=\left[\hat{H}_{1}, \hat{H}_{3}, \ldots, \hat{H}_{61}\right]^{T}$. Or $\hat{m}_{b}$ can be detected through (4) with data of the next half frame. Finally $m_{0}$ and $m_{1}$ are obtained according to:

$$
\left\{\begin{array}{l}
\hat{m}_{0}=\min \left(\hat{m}_{a}, \hat{m}_{b}\right) \\
\hat{m}_{1}=\max \left(\hat{m}_{a}, \hat{m}_{b}\right)
\end{array}\right.
$$

where $\min (*)$ and $\max (*)$ represent minimum and maximum value respectively. Because of the similarity of (4) and (5), it is sufficient to analyze the detection performance of $\hat{m}_{a}$ without loss of generality.

\section{COHERENT DETECTION PERFORMANCE}

Define $\Gamma_{m}=\sum_{i=0}^{30}\left\{\operatorname{Re}\left[\mathbf{Z} 0_{i}-\mathbf{s}_{i}^{m} \hat{\mathbf{H}}_{0, i}\right]\right\}^{2}, m \in\{0,1, \ldots 30\} \quad$, and the error probability of $\hat{m}_{a}$ can be expressed as

$$
\begin{aligned}
P_{e}= & P[\underbrace{\left(\Gamma_{t}>\Gamma_{0}\right) \cup\left(\Gamma_{t}>\Gamma_{1}\right) \cup \cdots \cup\left(\Gamma_{t}>\Gamma_{30}\right)}_{30}] \\
= & \sum_{\substack{i=0 \\
i \neq t}}^{30} P\left(\Gamma_{t}>\Gamma_{i}\right)-\sum_{\substack{i=0 \\
i \neq j \\
i \neq t}}^{30} \sum_{j=0}^{30} P\left[\left(\Gamma_{t}>\Gamma_{i}\right) \cap\left(\Gamma_{t}>\Gamma_{j}\right)\right]+ \\
& \sum_{\substack{i=0 \\
i \neq j \\
i \neq k \\
i \neq k \\
i \neq t \\
j \neq k}}^{30} \sum_{\substack{j \neq t \\
k \neq t}}^{30} \sum_{\substack{k=0 \\
k \neq t}}^{30} P\left[\left(\Gamma_{t}>\Gamma_{i}\right) \cap\left(\Gamma_{t}>\Gamma_{j}\right) \cap\left(\Gamma_{t}>\Gamma_{k}\right)\right]- \\
& \cdots-P\left[(\underbrace{\left(\Gamma_{t}>\Gamma_{0}\right) \cap\left(\Gamma_{t}>\Gamma_{1}\right) \cap \cdots \cap\left(\Gamma_{t}>\Gamma_{30}\right)}_{30}]\right.
\end{aligned}
$$

where $t$ is the target value.

The flat fading channel $H$ can be simulated by a first order autoregressive (AR1) model [9]

$$
H(k)=\alpha H(k-1)+\bar{\alpha} W 1(k)
$$

where $\bar{\alpha}=\sqrt{1-\alpha^{2}} \quad, \quad W 1(k) \sim \mathcal{C} N(0,1)$. There is $\alpha=J_{0}\left(2 \pi f_{d} \tau\right)$ in a Jakes model, where $J_{0}(*), f_{d}$ and $\tau$ are the zeros order of the first kind Bessel function, the maximum Doppler frequency offset and channel time interval respectively. Assume that channel estimation error follows Gaussian distribution [10], and then channel estimation error can be expressed as:

$$
\begin{aligned}
\hat{H}(k) & =H(k)+W 2(k)=\alpha H(k-1)+\overline{\alpha W 1}(k)+W 2(k) \\
& =\alpha H(k-1)+\beta W(k)
\end{aligned}
$$

where $W 2(k) \sim \mathcal{C N}\left(0, \delta^{2}\right), W 1(k)$ and $W 2(k)$ are independent of each other. Accordingly, there is $W(k) \sim \mathcal{C} N(0,1) \quad$ with $\beta=\sqrt{1-\alpha^{2}+\delta^{2}}$. Define $Q_{m}=\Gamma_{m}-\Gamma_{t}, m \neq t$, then 


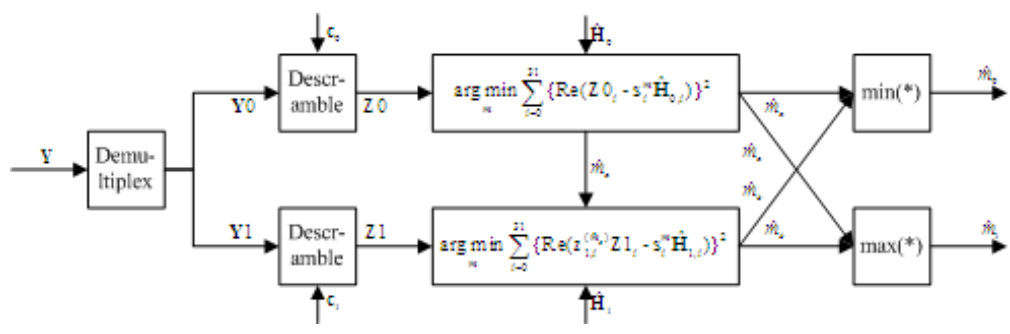

Figure 1. Coherent detection structure

$$
\begin{aligned}
Q_{m}= & \sum_{i=0}^{30}\left[\operatorname{Re}\left(H \mathbf{s}_{i}^{t}+\mathbf{N} 3_{i}-\left(\alpha H+\beta W_{i}\right) \mathbf{s}_{i}^{m}\right)\right]^{2} \\
& -\sum_{i=0}^{30}\left[\operatorname{Re}\left(H \mathbf{s}_{i}^{t}+\mathbf{N} 3_{i}-\left(\alpha H+\beta W_{i}\right) \mathbf{s}_{i}^{t}\right)\right]^{2} \\
= & \sum_{\mathbf{s}_{i}^{t} \neq \mathbf{s}_{i}^{m}} 4\left[\alpha \operatorname{Re}(H)^{2} \pm \alpha \operatorname{Re}(H) \operatorname{Re}\left(\mathbf{N} 3_{i}\right)\right. \\
& \left.+\beta \operatorname{Re}(H) \operatorname{Re}\left(W_{i}\right) \pm \beta \operatorname{Re}\left(W_{i}\right) \operatorname{Re}\left(\mathbf{N} 3_{i}\right)\right]
\end{aligned}
$$

(10) utilizes the following property:

$$
\left\{\begin{array}{l}
\mathbf{s}_{i}^{t}-\mathbf{s}_{i}^{m}= \pm 2, \mathbf{s}_{i}^{m} \times \mathbf{s}_{i}^{t}=-1, \quad \mathbf{s}_{i}^{t} \neq \mathbf{s}_{i}^{m} \\
\mathbf{s}_{i}^{m} \times \mathbf{s}_{i}^{m}=1, \quad \mathbf{s}_{i}^{t} \times \mathbf{s}_{i}^{t}=1
\end{array}\right.
$$

Define $S_{x y}=\left\{\mathbf{s}_{i}^{x} \neq \mathbf{s}_{i}^{y} \mid i=0,1, \ldots, 30 ; x \neq y\right\}$, it can be seen from [2] that

$$
\left|S_{x y}\right|=16,\left|S_{x y} \cap S_{x z}\right|=8
$$

where $|*|$ is the cardinality of a set. (12) means that there are 16 different values on the corresponding subcarriers between any two different $\mathbf{s}^{x}$ and $\mathbf{s}^{y}$, and there are 8 same values on the corresponding subcarriers between the two sets formed by any three different sequences. And (10) can be changed into

$$
Q_{m}=64 \alpha \operatorname{Re}(H)^{2}+X_{m}
$$

where $\quad X_{m}=\sum_{16} 4\left[ \pm \alpha \operatorname{Re}(H) \operatorname{Re}\left(\mathrm{N}_{i}\right)+\beta \operatorname{Re}(H) \operatorname{Re}\left(W_{i}\right) \pm\right.$ $\left.\beta \operatorname{Re}\left(W_{i}\right) \operatorname{Re}\left(\mathbf{N}_{i}\right)\right]$. Using central limit theorem [11], $Q_{m}$ follows Gaussian distribution with $Q_{m} \sim N\left(64 \alpha \operatorname{Re}(H)^{2}\right.$ , $\left.128 \alpha^{2} \operatorname{Re}(H)^{2} \sigma^{2}+128 \beta^{2} \operatorname{Re}(H)^{2}+64 \beta^{2} \sigma^{2}\right)$. The cross covariance between any $Q_{i}$ is $K_{Q_{i} Q_{j}}=64 \alpha^{2} \operatorname{Re}(H)^{2} \sigma^{2}+$ $64 \beta^{2} \operatorname{Re}(H)^{2}+32 \beta^{2} \sigma^{2}, i \neq j$.

So $\mathbf{Q}=\left[Q_{i}, Q_{j}, \ldots, Q_{n}\right]_{n}^{T}$ follows Gaussian distribution with mean vector $\quad \mathbf{m}=\left[64 \alpha \operatorname{Re}(H)^{2}, 64 \alpha \operatorname{Re}(H)^{2}, \ldots\right.$, $\left.64 \alpha \operatorname{Re}(H)^{2}\right]_{n}^{T}$, and the element of covariance matrix $\mathbf{K}$ can be express as:

$$
\mathbf{K}_{i j}=\left\{\begin{array}{l}
128 \alpha^{2} \operatorname{Re}(H)^{2} \sigma^{2}+128 \beta^{2} \operatorname{Re}(H)^{2}+64 \beta^{2} \sigma^{2}, i=j \\
64 \alpha^{2} \operatorname{Re}(H)^{2} \sigma^{2}+64 \beta^{2} \operatorname{Re}(H)^{2}+32 \beta^{2} \sigma^{2}, \quad i \neq j
\end{array}\right.
$$

So (7) can be simplified as:

$$
P_{e}=\sum_{n=1}^{30}(-1)^{n+1} C_{30}^{n} P(\underbrace{Q_{i}<0, Q_{j}<0, \ldots, Q_{n}<0}_{n})
$$

\section{SIMULATIONS}

The simulated LTE system has one transmitting antenna and one receiving antenna, the central frequency is $2.6 \mathrm{GHz}$. The Rayleigh channel is generated through Jakes model with a block fading characteristic, which means that the channel is unchanged during the span of an OFDM symbol. The simulation tool is Matlab which has an error in calculating high dimension Gaussian probability due to its calculation limit. Approximate value is calculated through the following formula with $1 \leq M \leq 30$ instead of (15) in order to decrease computational error.

$$
P_{e} \geq \sum_{n=1}^{M}(-1)^{n+1} C_{M}^{n} P(\underbrace{Q_{i}<0, Q_{j}<0, \ldots, Q_{n}<0}_{n})
$$

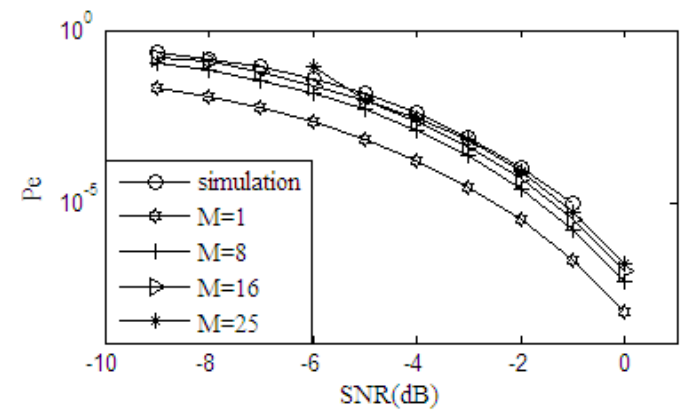

Figure 2. $P_{e}$ with different $M$ moving at 0

Figure 2. shows the theoretical value of (16) with different $M$ and simulation result with ideal channel estimation when the moving speed is 0 . It is shown that theoretical value is approaching the simulation result along 
with the increase of $M$, and it has a good approximation with $M=16$. Because of computational error, the theoretical result jumps at $\mathrm{SNR}=-6 d B$ and even goes negative at lower SNR. Accordingly, $M=16$ is adopted to calculate the theoretical result in the following simulations. Figure 3. shows the theoretical and simulated results of different systems with ideal channel estimation moving at $200 \mathrm{~km} / \mathrm{h}$. The theoretical results are consistent with the simulated results. It can also be seen that Doppler Effect has more influence on the TDD system than that on FDD system due to its bigger time span between PSS and SSS.

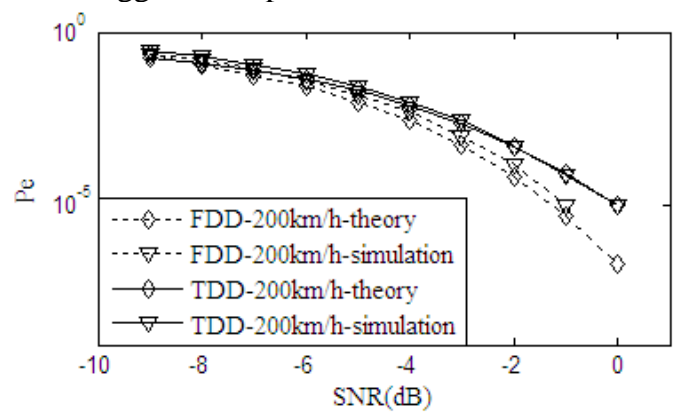

Figure 3. $P_{e}$ of FDD and TDD system moving at $200 \mathrm{~km} / \mathrm{h}$

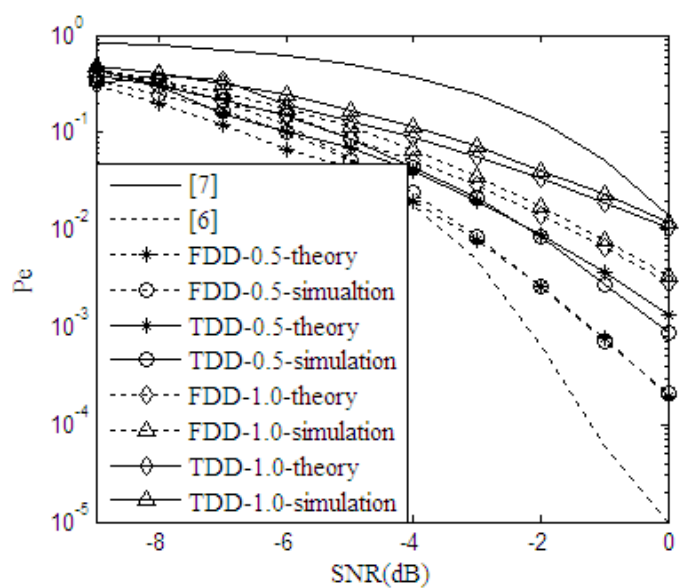

Figure 4. $\quad P_{e}$ of different methods with different channel estimation error moving at $200 \mathrm{~km} / \mathrm{h}$

Figure 4. shows the detection error probability of different methods with different channel estimation errors moving at $200 \mathrm{~km} / \mathrm{h}$. The compared algorithms are the differential detection in [7] and non-coherent detection in [6] with one segmentation. The theoretical and simulated results of coherent detection with different channel estimation error are consistent with each other. The detection performance is becoming worse along with the increase of error variance of channel estimation. Chanel estimation error has more influence on the performance of TDD than that of FDD. Algorithm in [6] has a better performance than coherent detection method when SNR is larger than $-4 \mathrm{~dB}$. Although algorithm in [7] eliminates the influence of channel attenuation, it introduces the multiplex of noise item, which deteriorates the detection performance. Accordingly, a detection structure with two kinds of detection methods can be exploited in a practical system. The alternate criterion is SNR, channel estimation error and system type correlated.

\section{CONCLUSIONS}

The Doppler Effect and channel estimation error are properly modeled, based on which the non-ideal SSS coherent detection performance is deduced. The theoretical result is approved through simulations. By comparing the differential, non-coherent and coherent detection methods with different channel estimation error, it can be seen that differential detection is always worse and non-coherent detection is better than coherent when SNR is larger than a threshold. Accordingly, it is suggested that a hybrid detection structure be adopted in a practical system.

\section{REFERENCES}

[1] S. Sesia, I. Toufik, M. Baker, "LTE-The UMTS long term evolution: Form theory to practice,” New YorkL John Wiley \& Sons, 2009, pp. $1-3$.

[2] 3GPP TS 36.211 v10.4.0., "Technical specification group radio acess network: Evoved universal terrestrial radio acess, physical channels and modulation (Release 10),” 2011.

[3] Baojin Li, Gang Sun, Xin Wang, "SSS Detection scheme for neighbor cell search in 3GPP LTE systems,” GLOBCOM, 2011, pp. 1-5.

[4] Baojin Li, Xin Wang, "Efficient SSS detection for neighbor cell Search in 3GPP LTE TDD systems,” ICC, 2011, pp. 1-5.

[5] Feng Wang, Yu Zhu, Zongxin Wang, “A low complexity scheme for S-SCH detection in 3GPP LTE downlink system," Global Mobile Congress 2010, pp. 1-6.

[6] Jung-In Kim, Jung-Su Han, Hee-Jin Roh, Hyung-Jin Choi, “SSS detection method for initial cell search in 3GPP LTE FDD/TDD dual mode receiver ," International Symposium on Communications and Information Technology, 2009, pp. 199-203.

[7] Chia-Chun Liao, Pei-Yun Tsai, Tzi-Dar Chiueh, "Low-complexity cell search algorithm for interleaved concatenation ML-sequences in 3GPP-LTE systems,” IEEE Wireless Communication Letters,” 2012, PP(99), pp. 1-4

[8] Pei-Yun Tsai, Hsiang-Wei Chang, "A new cell search scheme in 3GPP long term evolution downlink OFDMA systems,” Internal Conference on Wireless Communications \& Signal Processing, 2009, pp. 1-5.

[9] Li Ting Yang Lvxi, “A limited feedback precoding method based on adaptive subspace tracking and Jacobi algorithm for time-varying channels," Chinese High Technology Letters, 2009, 19(8), pp. 795799.

[10] D. Van Welden, F. Simoens, H. Steendam, M. Moeneclaey, "Impact of channel estimation errors on the performance of linear FIR equalizers for frequency selective MIMO channels," IEEE Symposium on Communications and Vehicular Technology, 2005, pp. 1-6.

[11] John G. Proakis, “Digital communications (4 ${ }^{\text {th }}$ edtion)," Beijing, Electronic Industry Press, 2008, pp. 56-61 